\title{
Neural Substrates of Reserve Observed in a Non-Demented Aging Population
}

\author{
Jeanyung Chey ${ }^{1,2 *}$, M Justin Kim ${ }^{3}$, Yaakov Stern², Minyoung Shin ${ }^{1}$, Hong-Sik Byun ${ }^{4}$ and Christian Habeck ${ }^{2}$
}

${ }^{1}$ Department of Psychology and Interdisciplinary Program for Brain Sciences, Seoul National University, Seoul 151-746, South Korea ${ }^{2}$ Cognitive Neuroscience Division, Department of Neurology, Columbia University College of Physicians and Surgeons, New York, NY 10032, USA ${ }^{3}$ Department of Psychological and Brain Sciences, Moore Hall, Dartmouth College, Hanover, NH 03755, USA

${ }^{4}$ Department of Radiology, Samsung Medical Center, Sungkyunkwan University School of Medicine, Seoul 135-710, South Korea

\begin{abstract}
Cognitive decline in dementia does not correspond precisely to the amount of neurodegeneration in the brain. This discrepancy in brain damage and its clinical manifestation has been explicated by the concept of reserve. Brain reserve inferred from the brain size had moderate success in explaining the discrepancy, and numerous studies have reported the effects of education supporting cognitive reserve. Yet the neural substrates of reserve have been elusive. Utilizing optimized voxel-based morphometry, we have identified brain regions that were significantly smaller in individuals with low cognitive performance (LCP) compared to those with normal cognitive performance (NCP) in community-residing non-demented elderly people both with low educational attainment. It was assumed that the cognitive performance in this population reflected long-standing cognitive functioning of the individual, possibly the reserve, based on their stable follow-up performance and clinical interviews. Bilateral precuneus, right superior frontal gyrus and left middle frontal gyrus were smaller in individuals with LCP. Further, the LCP individuals had weaker correlation between the gray matter volume of those regions and the rest of the cortex. On the other hand, volume of these regions was more tightly correlated with the K-DRS Total score in these individuals. Finally, an outcome study of the community sample from which this study's participants were recruited from reported five times higher risk of dementia in the LCP group. Precuneus and prefrontal cortex are proposed as key sites comprising the neural substrates that underlie the reserve.
\end{abstract}

Keywords: Education; Precuneus; Prefrontal cortex; Structural correlation; Voxel-based morphometry

Individuals with superior intelligence develop dementia symptoms later in the course of illness and low education has been repeatedly observed as a risk for Alzheimer's disease and dementia in general [1-3]. It has been proposed that individuals with greater cognitive reserve, measured with proxies such as education, occupation, and the Intelligence Quotient (IQ), can withstand the effects of brain injury or neurodegenerative processes and delay the clinical manifestation of dementia, while individuals with smaller reserve would manifest the symptoms earlier in the disease process $[2,4,5]$.

Reserve refers to the individual's ability to tolerate the effect of brain disease, and delay or even prevent the development of clinical symptoms of neuropathology [2,5]. Initially, reserve was conceptualized in simple brain measures of integrity, such as the whole brain volume and synaptic density $[1,6]$, characterized as brain reserve [2]. Conceptualized it cognitively as active a cognitive process that enables the individual to cope with after brain damage or neurodegeneration. Consistent with the reserve theory, education, the most popular proxy, has been repeatedly identified as a moderator in the development of dementia in longitudinal outcome studies. While low educational attainment has been found to be a significant risk factor for dementia [7-9], greater years of education has been shown to delay the clinical manifestation of dementia in proportion to the number of years $[4,10]$. These findings were confirmed and replicated in post-mortem neuropathology [11-13] as well as neuroimaging studies [14-17]. Occupation, intelligence and more recently leisure activities, other proxies of cognitive reserve, have also shown protective effects against dementia [5].

Recently, attempts to investigate the neural mechanism underlying cognitive reserve have been reported. A study found a common neural network involving the superior and medial frontal gyri that was activated as the processing load increased in a delayed item-recognition task. Based on the assumption that cognitive reserve would share brain network activated during demanding processing load, the results suggest that this network may be consistent with a neural representation of the cognitive reserve [18].

Brain maintenance during aging is another factor that could account for individual differences in dementia risk [19] and studies have shown that elderly individuals with minimal cognitive aging tended to have less senescent or pathological changes in their brain. Prefrontal cortex (PFC), the brain region showing earliest signs of aging, has been studied extensively to examine brain maintenance. For instance, PFC has been found to be thicker in elderly people who performed better in the Wisconsin Card Sorting Test, a classical test for higher cognitive functioning [20]. Further, successful brain maintenance in this region was manifested in high-performing elderly people who recruited cortical networks similar to young subjects during a working memory task $[21,22]$.

Compared to the neural correlates of cognitive reserve or the brain maintenance factors, the features of brain reserve have been elaborated in scant detail. The earlier theories of brain reserve $[1,6]$ focusing on whole brain volume or the synaptic density were effective in explaining

*Corresponding author: Jeanyung Chey, Department of Psychology, Seoul National University, Building 16, Gwanakro 599, Seoul 151-746, South Korea, Tel: +822-880-6432; E-mail: jychey@snu.ac.kr

Received December 07, 2016; Accepted December 26, 2016; Published January 02, 2017

Citation: Chey J, Justin Kim M, Stern Y, Shin M, Byun HS, et al. (2016) Neural Substrates of Reserve Observed in a Non-Demented Aging Population. J Alzheimers Dis Parkinsonism 6: 294. doi: 10.4172/2161-0460.1000294

Copyright: (c) 2016 Chey J, et al. This is an open-access article distributed under the terms of the Creative Commons Attribution License, which permits unrestricted use, distribution, and reproduction in any medium, provided the original author and source are credited. 
the reserve effect by utilizing a quantitative threshold [23] and studies have demonstrated that gross brain volumes and IQ, a key proxy of the reserve, were correlated in young [24] as well as old adults [25]. The brain size, however, is limited in accounting for the individual difference in cognitive processing that could make a significant difference within similar brain-sized people [2]. And it has been shown that fMRI activation patterns during a visual encoding task correlated better with the proxies such as IQ, education, occupation, and leisureactivities [26] than the whole brain volume. Further, brain reserve has been considered a passive model due to its assumption of a threshold at which clinical symptoms manifest without consideration of individual differences in how brain processes cognitive tasks [2]. While this may have been a valid point, recent studies have found evidence that brain structures as well as functions change over the adult lifespan due to developmental [27,28], environmental $[29,30]$ and medical factors [31]. Further, whole brain volume is a single index that does not reflect individual differences in regional structures. Since recent structural imaging techniques provides voxel counts, volume or thickness of the regions, the brain reserve concept can be approached anew using these regional measures, and shed light into the neural substrates of cognitive differences in elderly people. Studies have investigated the relation between intelligence and regional brain measures mostly in younger adults, and found cortical regions, mostly the prefrontal cortex and the parietal lobe [32] to be associated with IQ. Yet, few studies have explored the regional brain volumes that are associated with cognitive ability or proxies of reserve in the context of aging.

In this study, we attempted to find brain regions that were associated with overall cognitive functioning in non-demented communityresiding elderly people. We analyzed the regional gray matter volume of 13 individuals with low cognitive performance (LCP) and compared them to those with 13 normal cognitive performance (NCP) who was matched in age and education. They were all recruited from a sample of 243 non-demented community-residing elderly Koreans who were followed-up longitudinally for 6 years and had been evaluated for dementia diagnosis [33]. Since the cognitive performance in this sample remained stable and did not decline during the following four years, it was assumed that cognitive performance in this population at baseline measures individual's long-standing cognitive functioning status.

\section{Materials and Methods}

\section{Participants}

Twenty six individuals were recruited from a sample of 243 nondemented community-residing elderly people in the Seoul National University Cognitive Aging Study (SNU-CAS) at baseline. Individuals in the SNU-CAS were non-demented and "normal" according to a criterion from a previous study [34]. They 1) were free of memory and cognitive disorders leading to corresponding functional impairments in everyday life; 2) lived independently without difficulty; 3) had no psychiatric and neurological illness; and 4) had no physical conditions that are known to compromise cognitive capacity such as hypertension or diabetes that could not be controlled by medication, thyroid disease or loss of consciousness for more than $20 \mathrm{~min}$. The participants did not have severe hearing or vision problems that could significantly compromise the neuropsychological test results.

A battery of neuropsychological tests, clinical tests, and interviews were administered to assess the cognitive and behavioural functions that were necessary to ascertain the "normal" status of the elderly participants. Neuropsychological assessment involved 1) a neuropsychological interview with the subject, 2) the Korean version of the Dementia Rating Scale [35,36], 3) the Elderly Memory disorder Scale [37], 4) the Beck Depression Inventory [38], 5) the Edinburgh Handedness Inventory [39], 6) the Literacy Questionnaire [40] and a semi-structured interview [41] with an informant to confirm lack of significant change in cognitive and behavioural functions. Further details of the tools are in [42]. Therefore, no one with complaints of cognitive decline either from the subject him/herself or family members was included in the study.

Thirteen individuals had low cognitive performance (LCP) while thirteen had normal cognitive performance (NCP) according to the following criteria. LCP was defined by poor or borderline performance on either the Total score on the K-DRS or the Long-term Memory Recall score on the EMS; i.e., scores less than one standard deviation below the mean compared to the age- and education-specified norms. In order to be classified as having NCP, both the Total K-DRS and the Long-Term Memory Recall of the EMS had to be equal or above the cut-off score.

The two groups were matched on age and years of education, but were significantly different in the rate of literacy (Table 1). The subjects also received baseline brain MRI for voxel-based morphometry. These individuals had no significant findings on their T1 and T2 weighted MRI images that could suggest brain disorders or lesions [42] for detailed description on the exclusionary criteria.

\section{Procedures}

At baseline, each participant received an oral briefing on the nature of the study and signed an agreement of participation, followed by a comprehensive neuropsychological evaluation and sampling of the venous blood sample for the determination of the ApoE genotype. Lastly, brain magnetic resonance imaging (MRI) scans from thirtyone participants were obtained. Since the institutional review board of Seoul National University was not present during the time of the sevenyear study, researchers abided by the Declaration of Helsinki [43]. The procedural details of the baseline study are described in a previous study [42].

\section{Acquisition and analysis of the magnetic resonance image data}

Whole brain magnetic resonance images were acquired on a 1.5-T Signa system (General Electric Medical System, Milwaukee, WI, U.S.A). The MRI protocol included the following: (a) sagittal T1-weighted

\begin{tabular}{|c|c|c|c|}
\hline & $\begin{array}{l}\text { LCP }(n=13) \\
\text { Mean+SD or } \\
\text { frequency }\end{array}$ & $\begin{array}{l}\text { NCP }(n=13) \\
\text { Mean+SD or } \\
\text { frequency }\end{array}$ & $\begin{array}{c}\text { Statistical } \\
\text { Significance }\end{array}$ \\
\hline Age & $71.38(8.05)$ & $71.15(7.87)$ & 0.942 \\
\hline Gender (male/female) & $5 / 8$ & $3 / 10$ & 0.673 \\
\hline Education & $3.00(4.32)$ & $2.77(3.44)$ & 0.882 \\
\hline Literacy (n) & 7 & 0 & $0.005^{*+}$ \\
\hline K-DRS Total & $101.92(12.09)$ & $128.38(9.22)$ & $0.000^{* *}$ \\
\hline EMS DM recall & $1.18(0.56)$ & $1.88(0.44)$ & $0.002^{* *}$ \\
\hline Apo e4 allele $(n)^{a}$ & 2 & 0 & 0.199 \\
\hline Hypertension $^{\mathrm{b}}$ & $1 / 11$ & $2 / 13$ & NS \\
\hline Diabetes & $1 / 13$ & $0 / 13$ & NS \\
\hline $\operatorname{LOC}(<20 \mathrm{~min})^{\mathrm{c}}$ & $4 / 13$ & $1 / 13$ & NS \\
\hline Habitual smoking & $1 / 13$ & $3 / 13$ & NS \\
\hline
\end{tabular}

aData was not available for three subjects

'Data was not available for two subjects cDuration of loss of consciousness

Table1: Descriptive statistics of the LCP and the NCP participants. 
acquisitions (repetition time (TR) $400 \mathrm{~ms}$, echo time (TE) 11, number of excitations (NEX) 1) with field of view (FOV) of $24 \mathrm{~cm}, 5-\mathrm{mm}$ slice thickness, and matrix size of $256 \times 256$; (b) T2-weighted acquisitions (TR 3,000, TE 100, NEX 2) in the coronal planes perpendicular to the long axis of the hippocampus with section thickness of $3 \mathrm{~mm}$, FOV of $21 \mathrm{~cm}$ and matrix size of $256 \times 256$; (c) coronal T1-weighted (TR $30 \mathrm{ms,}$ TE 7, NEX 1, flip angle $45^{\circ}$ ) three-dimensional (3D) spoiled gradient acquisitions with 124 sections at $1.6 \mathrm{~mm}$ slice thickness, FOV of $21 \mathrm{~cm}$ and matrix size of $256 \times 192$.

To investigate the group differences in regional gray matter volume, optimized voxel-based-morphometry (VBM) was utilized. We adapted the optimized VBM methods from a previous study [44]. To briefly summarize, data from all 26 participants were used to construct a study-specific template image. Creating a template image tailored to our study was especially important given that typical template images are derived from a young adult sample (e.g. MNI152). Subsequently, the original whole brain images were segmented into gray and white matter images. Unconnected non-brain voxels (i.e., voxels not containing gray matter, white matter, or cerebrospinal fluid) were then extracted from these segmented images [45].

Gray matter images were then spatially normalized again, limiting any effects of non-brain voxels in the normalization process, thereby acquiring optimal spatial normalization of gray matter images. Then, optimized normalization parameters derived from the previous step were reapplied to the original whole brain images. They were spatially normalized and then segmented into gray matter, white matter, and cerebrospinal fluid. In addition, the Jacobian determinants, derived from spatial normalization, were applied to all gray matter images in order to acquire volume information from the voxels. Finally, all images were smoothed using a Gaussian kernel (full-width-half maximum=8 mm).

\section{Statistical analyses}

Independent $\mathrm{t}$-test, chi-square test and analysis of variance (ANOVA) were utilized to tests the group differences on basic demographic, cognitive and health measures and descriptive statistics were calculated.

\section{Voxel-based morphometry}

All smoothed images were assigned to either the LCP or the NCP group. Then, the contrasts $(\mathrm{LCP}<\mathrm{NCP}, \mathrm{LCP}>\mathrm{NCP})$ were obtained, using the analysis of covariance (ANCOVA) model, implemented in SPM2 with age, sex and intracranial volume (ICV) as covariates of no interest. ICV was measured with the reconstructed sagittal images of 5 $\mathrm{mm}$ thickness SPGR MRI data, details of which are described in [42]. A significance level of $\mathrm{p}<0.001$ (uncorrected) and $\mathrm{k}=100$ voxels was used to report VBM results.

\section{Structural correlation analysis}

To examine how relevant brain regions correlate with one another in terms of gray matter density, we employed a seed-based structural correlation analysis by extracting gray matter volume values from the 4 ROIs ("seeds") identified by the LCP $<\mathrm{NCP}$ contrast performed in the aforementioned ANCOVA model. To enable a brain wide analysis of structural correlation we used the AAL-template [46] to convert the structural images of participants into 116 regions of interest (ROIs). To remove the influence of several confounding variables, a multiple regression model that included age, sex and ICV was run on all seed values and on all 116 ROIs across subjects. The residuals of this regression were then correlated in the subsequent analysis. This allowed us to visualize brain regions that correlated with the seed regions across the whole brain while controlling for the effects of age, sex and ICV, hence identifying a structural correlation network for each seed region. Additionally, in order to assess group differences in the structural correlation patterns, we computed each of the 4 structural correlation networks separately for the LCP and the NCP groups and compared the networks with a permutation test (10,000 iterations). Given that there were 4 seeds and 2 groups, a total of 12 structural correlation networks (4 for each group plus 4 for all 26 participants) are reported here.

\section{Regional brain volumes and cognitive functions}

To investigate the cognitive functions associated with the four seeds, we performed correlation analysis between the regions and the neuropsychological performance scores, controlling for age, sex and ICV. To further investigate the association of regional brain volumes with overall cognitive functioning, we extended the analysis of the previous section: we used the K-DRS Total score as a "seed" and correlated it with the 116 regional ROIs, again controlling for age, sex, intracranial volume as covariates of no interest. Additionally, in order to assess the LCP versus NCP group differences in the correlation pattern between the K-DRS Total and the brain regions, we compared the correlation pattern with a permutation test (10,000 iterations).

\section{Results}

\section{Low cognitive performance and regional gray matter volumes}

The LCP group showed significantly smaller gray matter volume in bilateral precuneus and areas in the prefrontal cortex (Figure 1 and Table 2). The most notable difference between the two groups were found in bilateral precuneus, but regions in the right superior frontal gyrus (SFG) and the left middle frontal gyrus (MFG) were also significantly different. Medial temporal lobe structures, such as the hippocampus, did not show differences in gray matter density between the two cognitive performance groups, consistent with the results from the ROI-based volumetry study [42].

\section{Structural correlation networks}

Volumes in four areas that were found to be significantly different between the two cognitive groups, were designated as the "seeds," and further correlation analyses were conducted between the seeds and the rest of the brain regions. It is of note that correlation between these seeds was highly correlated. The correlation between the right and the left precuenus was 0.952 , the right precuneus and the right SFG 0.803 , the right precuneus and left MFG 0.782 , the left precuneus and the right SFG 0.760, the left precuneus and the left MFG.746 and the right SFG and the left MFG 0.663 . The coefficients were larger in the NCP group, reaching significant difference in correlations of the left MFG with the right precuneus $(p=0.018)$ and the left precuneus $(p=0.031)$ respectively. The group differences, however, did not survive the multiple-comparison corrections $(\mathrm{p}=0.05 / 6)$.

\begin{tabular}{|c|c|c|c|c|c|c|c|}
\hline \multirow[t]{2}{*}{ Brain Regions } & \multicolumn{3}{|c|}{$\begin{array}{c}\text { MNI } \\
\text { Coordinates }\end{array}$} & \multirow{2}{*}{$\begin{array}{c}\text { Number } \\
\text { of } \\
\text { Voxels }\end{array}$} & \multirow[t]{2}{*}{$\operatorname{LCP}(n=13)$} & \multirow[t]{2}{*}{$\operatorname{NCP}(n=13)$} & \multirow[t]{2}{*}{$\mathbf{t}$} \\
\hline & $\mathbf{x}$ & y & $\mathbf{Z}$ & & & & \\
\hline Precuneus (L) & -3 & -67 & 30 & 2150 & $0.4709 \pm 0.0726$ & $0.5644 \pm 0.0976$ & 5.26 \\
\hline Precuneus (R) & 6 & -75 & 22 & 1176 & $0.3817 \pm 0.0708$ & $0.4756 \pm 0.0988$ & 5.22 \\
\hline SFG (R) & 39 & 14 & 55 & 101 & $0.2340 \pm 0.0279$ & $0.2972 \pm 0.0523$ & 5.34 \\
\hline MFG (L) & -31 & 26 & 38 & 209 & $0.2296 \pm 0.0318$ & $0.3306 \pm 0.0836$ & 5.12 \\
\hline
\end{tabular}

SFG: Superior Frontal Gyrus; MFG: Middle Frontal Gyrus

Table 2: Brain regions that were smaller in the LCP individuals compared to the NCP individuals at baseline. 
Citation: Chey J, Justin Kim M, Stern Y, Shin M, Byun HS, et al. (2016) Neural Substrates of Reserve Observed in a Non-Demented Aging Population. J Alzheimers Dis Parkinsonism 6: 294. doi: 10.4172/2161-0460.1000294

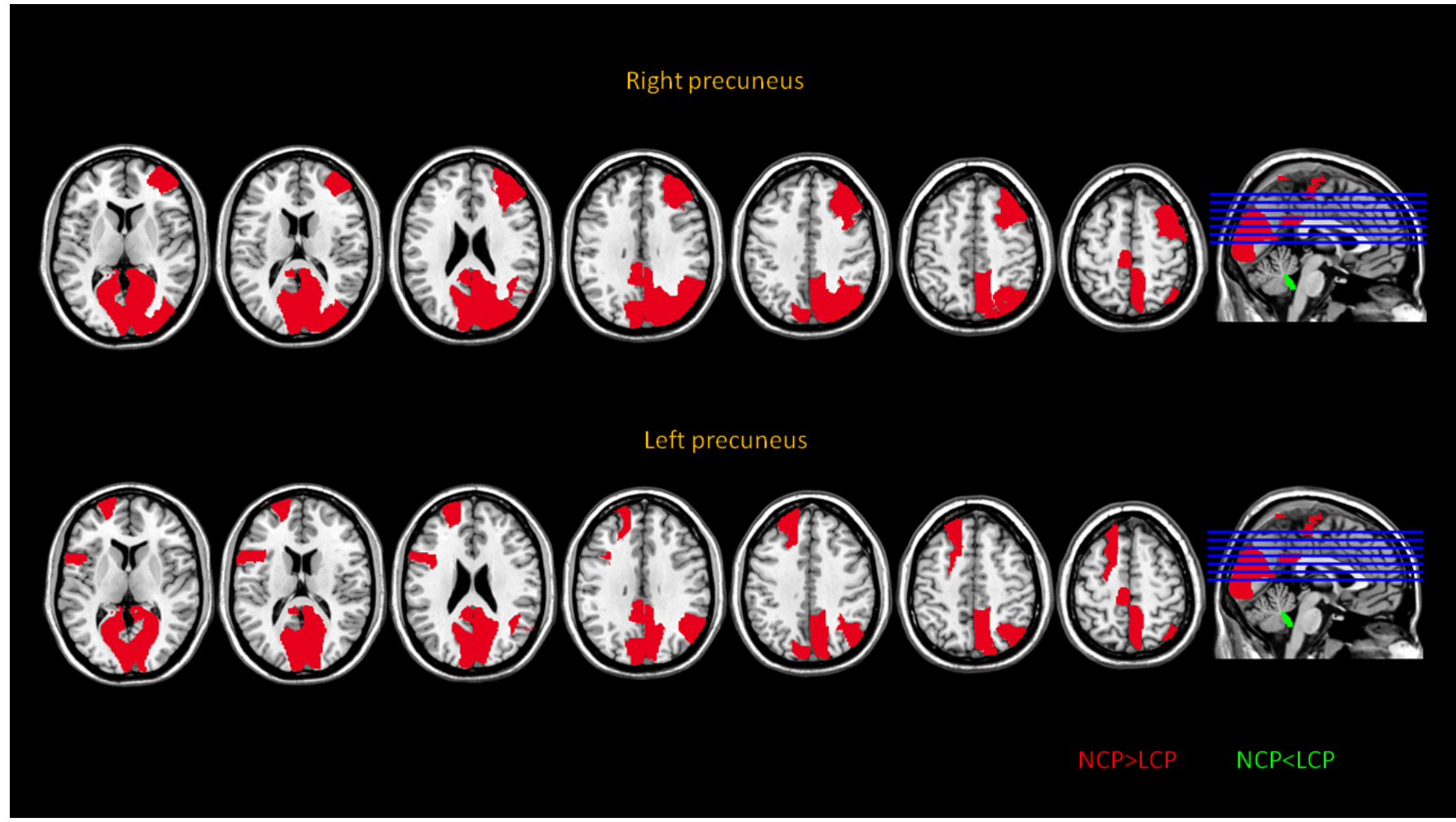

Figure 1: Brain regions significantly smaller in the LCP group compared to those in the NCP.

The structural correlation analysis between each of the four seeds and the 116 regions of interest (ROIs) were done to identify more comprehensive networks associated with the seeds and yielded four seed-based networks that are depicted in Figure 2. The precuneus-seeded network demonstrated significant correlations with lateral and medial frontal regions as well as medial parietal and occipital regions. Interestingly, the frontal seeds showed similar correlation patterns (Figure 2). We further compared the two cognitive performance groups, and found that correlation coefficients between cortical regions were generally greater in the NCP group (Table 3) for detailed results of the permutation tests.) The areas that showed significant group differences were found mostly in the precuneus-seeded networks. As depicted in Figure 3, the precuneus seeds had significantly higher correlation with the posterior cingulate cortex (PCC), the Calcarine cortex, right lingual gyrus and the paracentral lobule in the NCP group compared to the LCP. Similar difference was found with the right SFG-seeded network in the right Calcarine cortex, right lingual gyrus and the cuneus. The left MFG-seeded network showed group difference in the PCC and the left paracentral lobule. The significance of the group difference, however, did not survive the multiple-comparison correction $(\mathrm{p}<0.05 / 116)$.

\section{Brain regions and cognitive functions}

Many cognitive scores correlated significantly with the four seeds. As predicted, the total score of the K-DRS correlated significantly with the gray matter density of the areas (Table 4) for the correlation coefficients and top figure of Figure 4. Further, the correlation patterns between the K-DRS Total and the 116 ROIs in the two cognitive groups were investigated to find how brain regions were differently associated

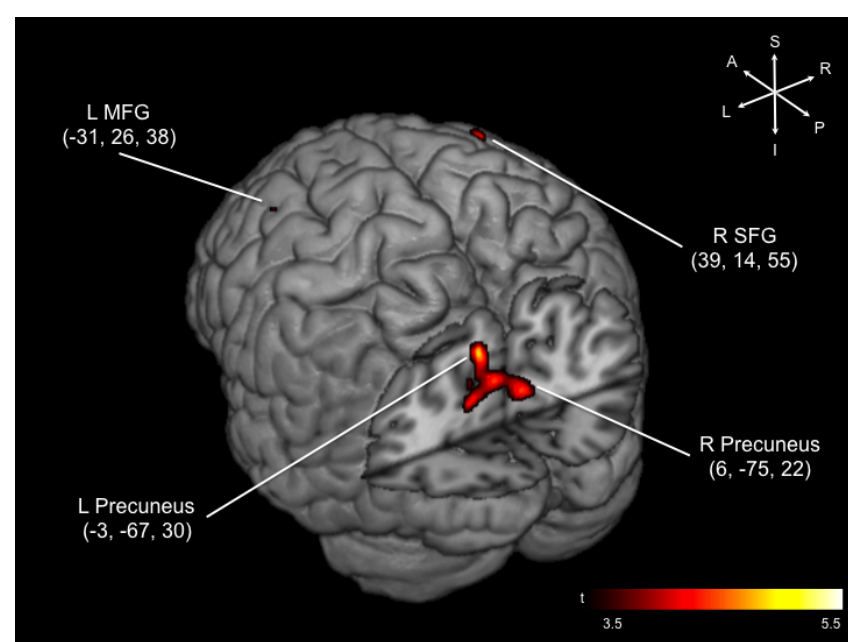

Figure 2: Structural correlation map between the four seeds and the $116 \mathrm{AAL}$ ROls across the combined group of LCP and NCP subjects. Only positive correlations were found at $p<0.05$.

with overall cognitive functioning. The K-DRS Total score and the gray matter density of the LCP individuals correlated more strongly than the NCP in the precuneus, the rectus, the vermis, the hippocampus, the frontal cortex, the posterior cingulate cortex and other cerebellar regions as shown in Figure 4. All the correlations were stronger in the LCP group. Scatter plots between the right precuneus, where the difference in correlation was the largest, and the K-DRS Total for both groups are shown in Figure 5. 
Citation: Chey J, Justin Kim M, Stern Y, Shin M, Byun HS, et al. (2016) Neural Substrates of Reserve Observed in a Non-Demented Aging Population. J Alzheimers Dis Parkinsonism 6: 294. doi: 10.4172/2161-0460.1000294

Page 5 of 9

\begin{tabular}{|c|c|c|c|c|c|c|c|}
\hline Precuneus (R) & & Precuneus (L) & & SFG (R) & & MFG (L) & \\
\hline AAL region & & AAL region & & AAL region & & AAL region & \\
\hline Mid Frontal, R & 0.047 & Sup Frontal, L & 0.043 & Calcarine, $\mathrm{R}$ & 0.0294 & PCC, L & 0.015 \\
\hline PCC, L & 0.004 & IFO, L & 0.046 & Cuneus, L & 0.0292 & PCC, R & 0.049 \\
\hline PCC, R & 0.007 & PCC, L & 0.014 & Cuneus, $\mathrm{R}$ & 0.0204 & Paracentral, L & 0.029 \\
\hline Calcarine, L & 0.027 & PCC, R & 0.008 & Lingual, $\mathrm{R}$ & 0.0472 & & \\
\hline Calcarine, $\mathrm{R}$ & 0.006 & Calcarine, L & 0.025 & & & & \\
\hline Cuneus, L & 0.036 & Calcarine, $\mathrm{R}$ & 0.005 & & & & \\
\hline Cuneus, $\mathrm{R}$ & 0.018 & Cuneus, L & 0.033 & & & & \\
\hline Lingual, $\mathrm{R}$ & 0.032 & Cuneus, $\mathrm{R}$ & 0.021 & & & & \\
\hline Sup Occipital, R & 0.042 & Angular, $\mathrm{R}$ & 0.011 & & & & \\
\hline Mid Occipital, R & 0.040 & Precuneus, $\mathrm{R}$ & 0.025 & & & & \\
\hline Inf Occipital, L & 0.038 & Paracentral, L & 0.023 & & & & \\
\hline Angular, $\mathrm{R}$ & 0.005 & Vermis 10 & -0.017 & & & & \\
\hline Precuneus, R & 0.034 & & & & & & \\
\hline Paracentral, L & 0.024 & & & & & & \\
\hline Vermis 10 & -0.048 & & & & & & \\
\hline
\end{tabular}

MFG: Middle Frontal Gyrus; SFG: Superior Frontal Gyrus; PCC: Posterior Cingulate Cortex; IFO: Inferior Frontal Operculum

Note the $p$-levels are sign-weighted with positive depicting LCP $<$ NCP negative LCP $>N C P$ and that only results at $p<0.05$ are listed

Table 3: AAL regions that showed significant difference in correlation with the seeds between the two cognitive performance groups.

\begin{tabular}{|c|c|c|c|c|}
\hline & Precuneus (L) & Precuneus (R) & SFG (R) & MFG (L) \\
\hline \multicolumn{5}{|l|}{ K-DRS } \\
\hline Total & $0.489^{*}$ & $0.435^{*}$ & $0.451^{*}$ & $0.567^{\star *}$ \\
\hline Attention & 0.398 & $0.427^{*}$ & $0.541^{* *}$ & $0.456^{*}$ \\
\hline Initiation/Perseveration & $0.442^{*}$ & 0.348 & 0.375 & $0.462^{* *}$ \\
\hline Construction & 0.044 & 0.035 & -0.021 & 0.183 \\
\hline Conceptualization & 0.353 & 0.341 & 0.266 & $0.510^{*}$ \\
\hline Memory & $0.432^{*}$ & 0.316 & 0.322 & 0.387 \\
\hline \multicolumn{5}{|l|}{ EMS } \\
\hline EMS LTM Recall & $0.515^{*}$ & $0.463^{*}$ & $0.466^{*}$ & $0.557^{* *}$ \\
\hline \multicolumn{5}{|l|}{ EVLT } \\
\hline Learning & $0.450^{*}$ & 0.355 & 0.370 & $0.513^{*}$ \\
\hline S-D Free Recall & $0.588^{* *}$ & $0.452^{*}$ & $0.429^{*}$ & 0.317 \\
\hline S-D Cued Recall & $0.562^{* *}$ & $0.458^{*}$ & $0.423^{*}$ & $0.518^{*}$ \\
\hline L-D Free Recall & $0.425^{*}$ & 0.409 & $0.453^{*}$ & $0.432^{*}$ \\
\hline L-D Cued Recall & $0.617^{* *}$ & $0.540^{* *}$ & $0.534^{* *}$ & $0.473^{*}$ \\
\hline Recognition & 0.264 & 0.209 & 0.329 & 0.261 \\
\hline \multicolumn{5}{|l|}{ SRFT } \\
\hline Copy & 0.304 & 0.368 & 0.283 & 0.372 \\
\hline Immediate & 0.233 & 0.311 & 0.241 & $0.419^{*}$ \\
\hline Delayed & 0.290 & 0.312 & 0.276 & $0.431^{*}$ \\
\hline \multicolumn{5}{|l|}{ Story Recall Test } \\
\hline Immediate & $0.468^{*}$ & 0.383 & $0.431^{*}$ & $0.429^{*}$ \\
\hline Delayed & $0.525^{*}$ & $0.429^{*}$ & $0.444^{*}$ & $0.500^{*}$ \\
\hline Recognition & 0.359 & 0.259 & 0.239 & 0.214 \\
\hline Clock Drawing Test (Total) & 0.405 & 0.351 & 0.286 & $0.455^{*}$ \\
\hline K-BNT (15 item) $)^{\star * *}$ & 0.152 & 0.093 & -0.109 & 0.054 \\
\hline Go-No-Go Test & 0.357 & 0.292 & 0.199 & 0.356 \\
\hline
\end{tabular}

${ }^{*} p<0.05,{ }^{* *} p<0.01$; MFG: Middle Frontal Gyrus; SFG: Superior Frontal Gyrus K-DRS: Korean-Dementia Rating Scale; EMS: Elderly Memory Disorder Scale; EVLT: Elderly Verbal Learning Test; SRFT: Simple Rey Figure Test; K-BNT: KoreanBoston Naming Test ( ${ }^{* * *}$ data were missing for four subjects; 1 NCP, 3 LCP)

Table 4: Partial correlation coefficients between neuropsychological measures and volumes of the key voxels controlled for age, sex and intracranial volume.

\section{Discussion}

\section{Neural substrates of reserve}

This study found that non-demented elderly individuals with low

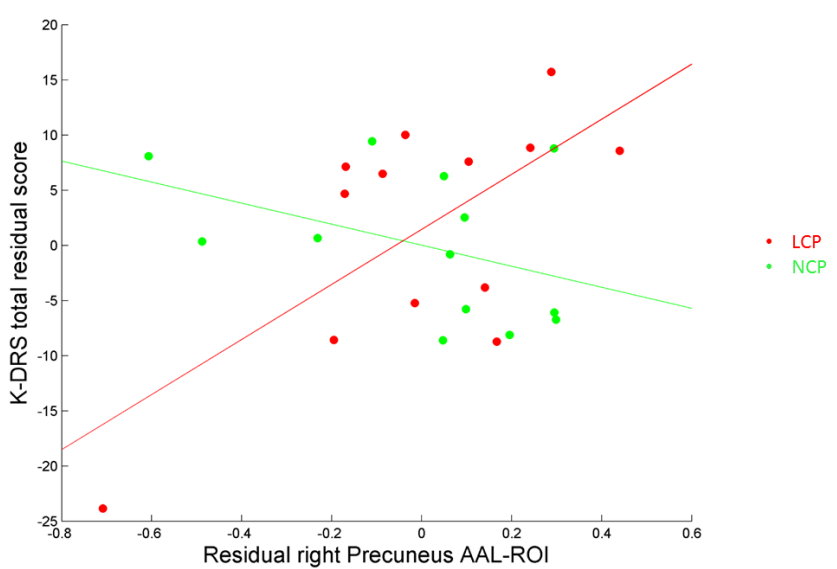

Figure 3: Significant differences in correlation in the two cognitive performance groups between the gray-matter density of $116 \mathrm{RO}$ (s and right and left precuneus, respectively, as ascertained by a permutation test with 10,000 precuneus, respectively, as ascertained by a permutation test with 10,000 correlations for the LCP, $p<0.05$.

cognitive performance (LCP) had significantly smaller gray matter volume or density in bilateral precuneus, the right superior frontal gyrus (SFG) and the left middle frontal gyrus (MFG) compared to those with normal cognitive performance. A subsequent correlation analysis with the four regions used as the seeds was conducted to further investigate the neural substrates underlying cognitive differences, which revealed an overlapping network of cortical regions that consisted of bilateral precuneus, the posterior cingulate cortex, paracentral lobule, the lateral and medial frontal regions, the angular gyrus and the medial and lateral occipital cortex. Moreover, the network differed significantly in its strength of correlation in the two cognitive performance groups; i.e., demonstrating greater correlation in the NCP group in the precuneusseeded networks which primarily consisted of the posterior cingulate cortex, the Calcarine cortex and the paracentral gyrus. Frontal seedednetworks showed the same pattern of greater correlation in the NCP group albeit with less significant regions. Lastly, the K-DRS Total score demonstrated association with the volume or density of the precuneus and this association was stronger in the LCP compared to 


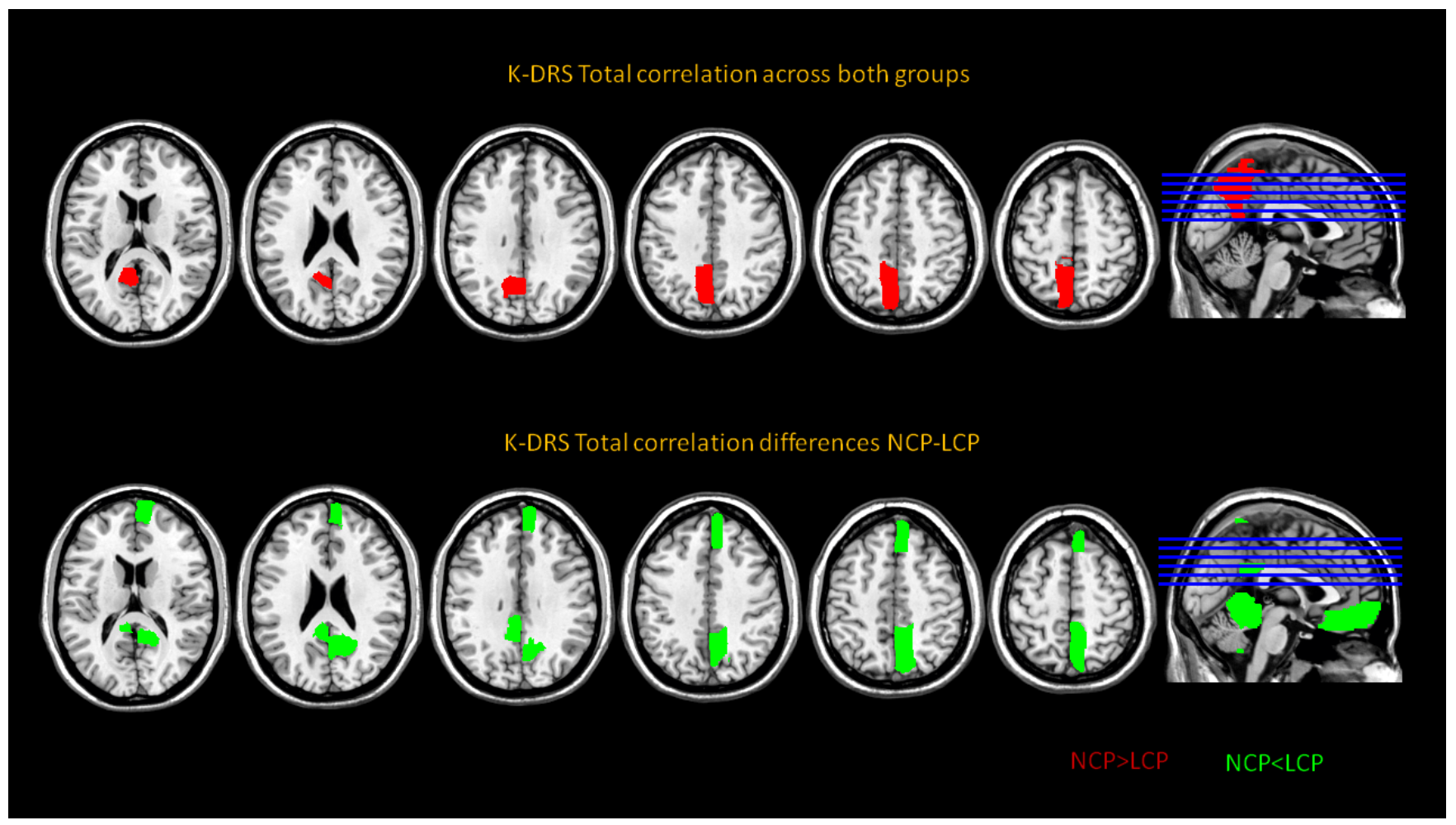

Figure 4: AAL brain regions whose gray-matter density correlated significantly with the K-DRS across both LCP and NCP groups (top). Regions that demonstrated significantly higher correlation between the volume and the KDRS Total score in the LCP group compared to the NCP as ascertained by a permutation test with 10,000 iterations. Green indicates larger correlations for the LCP, $p<0.05$ (bottom).

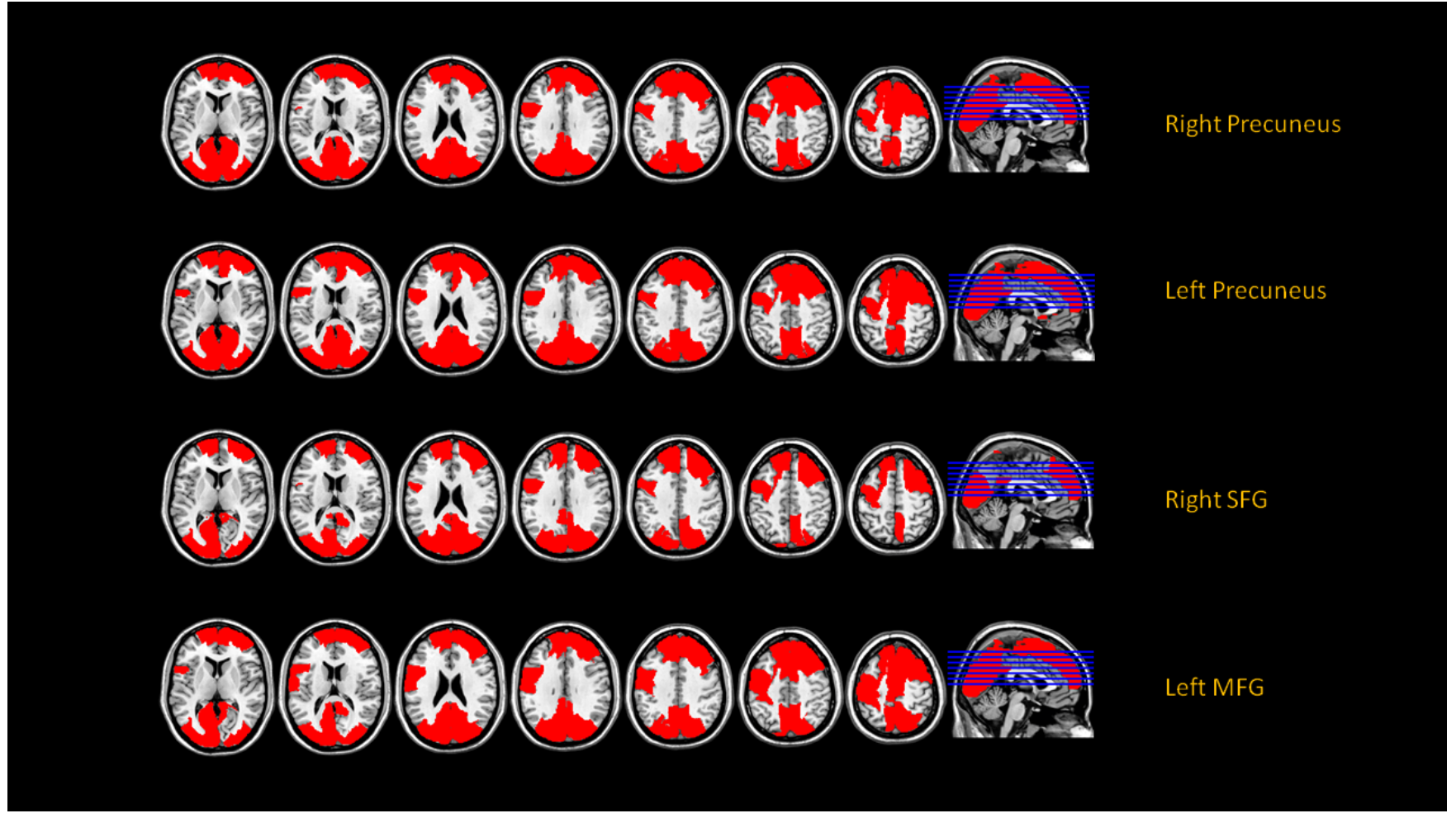

Figure 5: Scatter plots between the right precuneus volume and the K-DRS score in the LCP ( $r=0.666)$ and the NCP ( $r=-0.412)$ groups. The correlation coefficients of the two group differed significantly $(p=0.0076)$. 
the NCP group. This pattern of stronger correlation between the K-DRS Total score and the gray matter volume of various brain regions was observed in other brain regions, such as the orbital frontal cortex, the hippocampus, the posterior cingulum and the vermis of cerebellum.

\section{Nature of low cognitive performance}

It is unlikely that smaller regional gray matter volumes observed in the LCP group was due to mild cognitive impairment or preclinical dementia, since no LCP individuals showed decline, instead demonstrated improvement, in cognitive performance for 4 years following the initial assessment [47]. No atrophy was found in the medial temporal structures of the LCP individuals [42], which are often found to be smaller in individuals with mild cognitive impairment. Lastly, the subject recruitment procedure precluded elderly individuals with complaints of cognitive decline reported either by the individual him/herself or by the family member.

The true nature of lower cognitive performance in these individuals may be elusive, since we do not have access to their childhood and early adult cognitive performance. Nonetheless, several characteristics of the LCP individuals suggest that their cognitive performance reflects a longstanding cognitive functioning status possibly related to intelligence or cognitive reserve. First, a previous study found that intracranial volume (ICV) was significantly smaller in the LCP individuals compared to those of the NCP group [42], demonstrating that their brain reserve was smaller than the normal cognitive functioning individuals. But more importantly, all LCP individuals grew up in remote rural areas where educational opportunity was slim and not required for their livelihood. They were mainly farmers who all received equivalent or less than 6 years of schooling, a few not attending school at all. Since the NCP individuals were matched in terms of years of schooling, however, simple duration of education do not explain their low performance completely. Literacy, one of the basic outcomes of schooling, probably could add more information to the quality of educational experience elderly individuals had. Approximately half of the LCP individuals were functionally illiterate, while everyone in the NCP group was literate despite similar years of schooling. Therefore, the lack of opportunity or ability to learn to read or write could be one of the factors differentiating the two cognitive groups as observed in previous studies $[48,49]$. Since limited years of education was the necessary condition for all low cognitive performance in our sample, albeit not a sufficient one and literacy an additional dimension, it becomes clear that low cognitive performance in this population reflected life experience that was cognitive in nature. It appears that LCP individuals were not able to develop cognitive functions or brain to their full potential, most likely due to their limited educational experience. This is consistent with the finding that education narrows the variance of cognitive performance, especially boosting the performance of those in the lower strata [50]. We cannot, however, rule out the contribution of gene in cognitive performance. Since the two cognitive groups did not differ significantly in the apo e4 status, other genetic variations may have contributed to the cognitive differences in tandem with their educational experience.

\section{Strength of connectivity and cognitive performance}

Structural correlation analysis was able to further specify the neural substrates of cognitive difference in the elderly people; i.e., the reduced correlation in the seeded neural networks of the LCP elderly individuals compared to those of the NCP. The origin of this weaker connectivity or coherence of the cortical regions in LCP may be limited cognitive activities. A recent study examining the effects of education on brain metabolism reported findings supportive of this interpretation, showing that low educational attainment was associated with less functional connectivity especially in the long-distance connections [47], making them more vulnerable to both random and targeted attacks. The diminished cortical connectivity underlying low cognitive performance in the elderly individuals may be similar in nature, possibly making them more vulnerable to aging and neurodegeneration. In fact, elderly people with LCP in the larger community sample have been found to have significantly increased risk, i.e., five times greater chance of developing dementia seven years later, despite the fact that there was no decline in cognitive functions in the LCP elderly group during the initial four years of follow-up (Shin et al. [33], in preparation). In view of these results, we propose that a cortical network consisting of bilateral precuneus, the right frontal gyrus, and the left middle frontal gyrus could be one of the key brain regions that constitute the neural substrates of reserve.

\section{Correlation between precuneus volume and general cognition}

An interesting finding of this study is that a measure of general cognition, the K-DRS Total score, correlated with the seed regions more strongly in the LCP compared to the NCP, especially in the precuneus. This is consistent with a previous study that found head circumference predicted the risk of dementia only in nuns with low but not high educational attainment; i.e., individuals with smaller head circumference had greater chance of developing dementia only in the low education group. It appears that brain volume makes a greater difference in cognitive performance in individuals who lack or have low cognitive reserve, while it has limited effect in those with relatively high cognitive reserve. These findings of stronger association between brain size or volume and cognitive function are consistent with the cognitive reserve theory that proposes that individuals with higher reserve can cope with the pathology better via more efficient utilization of brain network [2], while those with low reserve will depend more on the size or the volume of the brain. More efficient utilization of the brain network assumed in the high cognitive reserve individuals could be mediated by stronger correlation or coherence of relevant cortical regions, as our findings suggest. Yet, the proposed cortical network was based on structural correlation of brain regions, which did not allow ascertaining the relationship between coherence and cognitive performance at an individual level. Studies investigating the functional connectivity from resting fMRI and its relation to cognitive function are needed to validate the proposed network.

\section{Nature of the precuneus and its associated network}

While the prefrontal cortex has been acknowledged as the brain region involved in many higher-cognitive functions [51], the role of precuneus in various cognitive functions has been revealed only more recently, mainly through functional neuroimaging studies [52]. Nestled in the medial portion of the posterior parietal lobe (BA 7 and 31) with its rich connection to association cortices and subcortical structures, it is activated during a host of tasks that require various cognitive functions, such as retrieval of episodic memory [53], visuospatial imagery [54], working memory [55] reasoning [56] and self-relevant processing $[57,58]$. More recent connectivity studies have also found that the precuneus is one of the key hubs in the human brain where longdistance connections as well as local connections are abundant both structurally [59] and functionally [60], thereby playing a strategically important role in various cognitive functions. In particular, the central precuneus, where the gray matter volume difference was found between the two cognitive performance groups, has been demonstrated to be structurally and functionally connected to areas involving associative 
and higher-cognitive functions, such as the inferior parietal and the dorsolateral prefrontal cortices as well as the medial temporal area [61]. Therefore, the precuneus appears to be one of the optimal brain regions that could underlie overall cognitive functioning differences, and contribute to the neural substrate of reserve, which would waive off the impact of neuropathology in dementias. The fact that precuneus regions showed the greatest difference in the structural correlation map between the two cognitive performance groups further supports this hypothesis.

In terms of aging, the frontal lobe, specifically the prefrontal cortex, is the most vulnerable brain area [62,63], diminishing its volume at the rate of five percent every decade since adulthood in human. On the other hand, many studies have identified the precuneus as one of the brain regions that are affected early in $\mathrm{AD}[64,65]$ or even in its prodromal phase [66,67]. It is also a region that has lower brain glucose metabolism in the mild cognitive impairment elderly people who later converted to AD [68]. Smaller volumes and weaker connectivity from these two brain regions are likely to make the brain vulnerable to the effects of aging and degenerative changes, further supporting their role in moderating the development of dementia.

\section{Summary and Caveats}

Limitations of the study must be addressed. Although this study was part of a six-year follow-up study, this study in itself was crosssectional, so the inference about the risk of dementia based on the community-sample is indirect and should be interpreted with caution. We recognize that the sample size was very small, and comparison of groups in the correlation analyses did not reach the corrected threshold for multiple comparisons. Future studies, therefore, with larger sample with different population are necessary. However, we would like to note that since the definition of LCP is based on normative data, they make up only $5-15 \%$ of the community-residing elderly population.

In summary, this study has found areas in the brain that are likely to be part of neural networks supporting brain reserve. Bilateral precuneus and its associated network was found to be less developed in elderly people with low cognitive performance compared to the normal performing counterpart, and these regional differences could mediate the risk of dementia in elderly individuals. Elucidating the neural substrates of reserve that are associated with cognitive performance would have implications for developing neuro-cognitive tools or programs for elderly people who are at greater risk for dementia due to low cognitive reserve.

\section{Acknowledgement}

The authors would like to thank Kyungmin Shin, Hoyoung Kim, Myungjin Kim, Hojung Song, and Sungha Lee for their indispensable help in collecting neuropsychological data, Jiae Cheong for proofreading the manuscript, Jong-Won Kim for his genotype analysis, Jason Steffener for his invaluable comments on the manuscript.

This work was supported by a grant (\#M103KV10021-03K2201-02130) from Brain Research Center of the 21st Century Frontier Research Program funded by the Ministry of Science and Technology of Republic of Korea, by the Brain Research Program (2015M3C7A1028376) through the National Research Foundation of Korea (NRF) funded by the Ministry of Science, ICT and Future Planning, and also by the 'Overseas Training Expenses for Humanities and Social Sciences' through Seoul National University (SNU) in 2014. The study abided by the Helsink Accords [43], and obtained informed consent from all the volunteers, and paid an honorarium for their participation. The Seoul National Unviersity Institutional Review Board was founded after the study was conducted. No actual or potential conflicts of interest were present in this study.

\section{References}

1. Katzman R, Aronson M, Fuld P, Kawas C, Brown T, et al. (1989) Development of dementing illnesses in an 80 year old volunteer cohort. Ann Neurol 25: 317 324

2. Stern $Y(2002)$ What is cognitive reserve? Theory and research application of the reserve concept. J Int Neuropsychol Soc 8: 448-460.

3. Valenzuela MJ, Sachdev P (2006) Brain reserve and cognitive decline: A nonparametric systematic review. Psychol Med 36: 1065-1073.

4. Brayne C, Ince PG, Keage HAD, McKeith IG, Matthews FE, et al. (2010) Education, the brain and dementia: Neuroprotection or compensation? EClipSE Collaborative Members. Brain 133: 2210-2216.

5. Fratiglioni L, Wang HX (2007) Brain reserve hypothesis in dementia. J Alzheimers Dis 12: 11-22.

6. Satz P (1993) Brain reserve capacity on symptom onset after brain injury: A formulation and review of evidence for threshold theory. Neuropsychology 7 : 273-295.

7. Letenneur L, Gilleron V, Commenges D, Helmer C, Orgogozo J, et al. (1999) Are sex and educational level independent predictors of dementia and Alzheimer's disease? Incidence data from the PAQUID project. J Neurol Neurosurg Psychiatry 66: 177-183.

8. Qiu C, Backman L, Winblad B, Aguero-Torres H, Fratiglioni L (2001) The influence of education on clinically diagnosed dementia incidence and mortality data from the Kungsholmen Project. Arch Neurol 58: 2034-2039.

9. Ravaglia G, Forti P, Maioli F, Martelli M, Servadei L, et al. (2005) Incidence and etiology of dementia in a large elderly Italian population. Neurology 64 1525-1530.

10. Hall C, Derby C, LeValley A, Katz M, Verghese J, et al. (2007) Education delays accelerated decline on a memory test in persons who develop dementia. Neurology 69: 1657-1664.

11. Bennett D, Schneider J, Wilson R, Bienias J, Arnold S (2005) Education modifies the association of amyloid but not tangles with cognitive function. Neurology 65: 953-955.

12. Koepsell TD, Kurland BF, Harel O, Johnson EA, Zhou XH, et al. (2008) Education, cognitive function, and severity of neuropathology in Alzheimer disease. Neurology 70: 1732-1739.

13. Roe C, Xiong C, Miller J, Cairns N, Morris J (2008) Interaction of neuritic plaques and education predicts dementia. Alzheimer Dis Assoc Disord 22: 188193.

14. Chiu N, Lee B, Hsiao S, Pai M (2004) Educational level influences regiona cerebral blood flow in patients with Alzheimer's disease. J Nucl Med 45: 18601863.

15. Kemppainen NM, Aalto S, Karrasch M, Någren K, Savisto N, et al. (2008) Cognitive reserve hypothesis: Pittsburgh Compound $B$ and fluorodeoxyglucose positron emission tomography in relation to education in mild Alzheimer's disease. Ann Neurol 63: 112-118.

16. Perneczky R, Drzezga A, Diehl-Schmid J, Schmid G, Wohlschläger A et al. (2006) Schooling mediates brain reserve in Alzheimer's disease: Findings of fluoro-deoxy-glucose-positron emission tomography. J Neurol Neurosurg Psychiatry 77: 1060-1063.

17. Stern Y, Alexander GE, Prohovnik I, Mayeux R (1992) Inverse relationship between education and parietotemporal perfusion deficit in Alzheimer's disease. Ann Neurol 32: 371-375.

18. Stern Y, Zarahn E, Habeck C, Holtzer R, Rakitin B, et al. (2008) A common neural network for cognitive reserve in verbal and object working memory in young but not old. Cereb Cortex 18: 959-967.

19. Nyberg L, Lövdén M, Riklund K, Lindenberger U, Bäckman L (2012) Memory aging and brain maintenance. Trends Cogn Sci 16: 292-305.

20. Burzynska AZ, Nagel IE, Preuschhof C, Gluth S, Bäckman L, et al. (2012) Cortical thickness is linked to executive functioning in adulthood and aging Hum Brain Mapp 33: 1607-1620.

21. Nagel IE, Preuschhof C, Li SC, Nyberg L, Bäckman L, et al. (2009). Performance level modulates adult age differences in brain activation during spatial working memory. Proc Natl Acad Sci U S A 106: 22552-22557.

22. Nagel IE, Preuschhof C, Li SC, Nyberg L, Bäckman L, et al. (2011) Load modulation of BOLD response and connectivity predicts working memory performance in younger and older adults. J Cogn Neurosci 23: 2030-2045. 
Citation: Chey J, Justin Kim M, Stern Y, Shin M, Byun HS, et al. (2016) Neural Substrates of Reserve Observed in a Non-Demented Aging Population. J Alzheimers Dis Parkinsonism 6: 294. doi: 10.4172/2161-0460.1000294

23. Schofield PW, Logroscino G, Andrews H, Albert S, Stern Y (1997)An association between head circumference and Alzheimer's disease in a population-based study of aging. Neurology 49: 30-37.

24. Andreasen NC, Flaum M, Swayze V 2nd, O'Leary DS, Alliger R, et al. (1993) Intelligence and brain structure in normal individuals. Am J Psychiatry 150: 130134

25. MacLullich AM, Ferguson KJ, Deary IJ, SeckI JR, Starr JM, et al. (2002) Intracranial capacity and brain volumes are associated with cognition in healthy elderly men. Neurology 59: 169-174.

26. Sole-Padulles C, Bartres-Faz D, Junque C, Vendrell P, Rami L, et al. (2009) Brain structure and function related to cognitive reserve variables in normal aging, mild cognitive impairment and Alzheimer's disease. Neurobiol Aging 30: $1114-1124$

27. Douaud G, Groves AR, Tamnes CK, Westlye LT, Duff EP, et al. (2014) A common brain network links development, aging and vulnerability to disease. Proc Natl Acad Sci U S A 111: 17648-17653.

28. Raz N, Lindenberger U, Rodrigue KM, Kennedy KM, Head D, et al. (2005) Regional brain changes in aging healthy adults: General trends, individual differences and modifiers. Cereb Cortex 15: 1676-1689.

29. Boyke J, Driemeyer J, Gaser C, Büchel C, May A (2008) Training-induced brain structure changes in the elderly. J Neurosci 28: 7031-7035.

30. Woollett K, Maguire EA (2011) Acquiring "the knowledge" of London's layout drives structural brain changes. Curr Biol 21: 2109-2114.

31. Raz N, Rodrigue KM, Acker JD (2003) Hypertension and the brain: Vulnerability of the prefrontal regions and executive functions. Behav Neurosci 117: 11691180.

32. Jung RE, Haier RJ (2007) The parieto-frontal integration theory (P-FIT) of intelligence: Converging neuroimaging evidence. Behav Brain Sci 30: 135-154.

33. Shin M, Lee S, Lee JY, Chey J (2016) Is low cognitive performance a risk for dementia in the elderly Korean population.

34. Hulette CM, Welsh-Bohmer KA, Murray MG, Saunders AM, Mash DC, et al. (1998) Neuropathological and neuropsychological changes in "normal" aging: evidence for preclinical Alzheimer disease in cognitively normal individuals. $J$ Neuropathol Exp Neurol 57: 1168-1174.

35. Chey J, Na DR, Park S, Park E, Lee S (1999) Effects of education in dementia assessment: Evidence from standardizing the Korean-dementia rating scale. Clin Neuropsychol 13: 293-302.

36. Mattis S (1988) Dementia rating scale. Psychological Assessment Resources Odessa, Fl.

37. Chey J (2007) Elderly memory disorder scale. Hakjisa, Seoul.

38. Hahn HM, Yum TH, Shin YW, Kim KH, Yoon DJ, et al. (1986) A standardization study of the Beck Depression Inventory in Korea. Korean J Neuropsychiatr 25: 487-500.

39. Oldfield RC (1971) The assessment and analysis of handedness: The Edinburgh inventory. Neuropsychologia 9: 97-113.

40. Moon H, Chey J (2004) Literacy and cognitive functions in the Korean elderly people. Korean J Geriatr Psychiatry 8: 112-120.

41. Sano M, DP D, Richards M, Miller LW, Marder K, et al. (1995) A standardized technique for establishing onset and duration of symptoms of Alzheimer's disease. Arch Neurol 52: 961-966.

42. Chey J, Na DG, Tae WS, Ryoo JW, Hong SB (2006) Medial temporal lobe volume of non-demented elderly individuals with poor cognitive functions Neurobiol Aging. 27: 1269-1279.

43. World Health Organization (1996) Declaration of Helsinki. BMJ 313: 1448-1449.

44. Kim MJ, Hamilton JP, Gotlib IH (2008) Reduced caudate gray matter volume in women with major depressive disorder. Psychiatry Res 164: 114-122.

45. Good CD, Johnsrude IS, Ashburner J, Henson RN, Friston KJ, et al. (2001) A voxel-based morphometric study of ageing in 465 normal adult human brains. Neuroimage 14: 21-36

46. Tzourio-Mazoyer N, Landeau B, Papathanassiou D, Crivello F, Etard O et al. (2002) Automated anatomical labelling of activations in spm using a macroscopic anatomical parcellation of the MNI MRI single subject brain Neuroimage 15: 273-289.
47. Kim Jl, Chey J, Kim H, Kim SE (2015) The effect of education on regional brain metabolism and its functional connectivity in an aged population utilizing positron emission tomography. Neurosci Res 94: 50-61.

48. Kim H, Chey J (2010) Effects of education, literacy and dementia on the Clock Drawing Test performance. J Int Neuropsychol Soc 16: 1138-1146.

49. Manly JJ, Touradji P, Tang MX, Stern Y (2003) Literacy and memory decline among ethnically diverse elders. J Clin Exp Neuropsychol 25: 680-690.

50. Chey J, Na DR, Park S, Park E, Lee S (1999) Effects of education in dementia assessment: Evidence from standardizing Korean-dementia rating scale. Clin Neuropsychol 13: 293-302.

51. Miller B, Cummings J (2007) The human frontal lobes: Functions and disorders (2nd edn) The Guilford Press, New York.

52. Cavanna AE, Trimble MR (2006) The precuneus: A review of its functional anatomy and behavioural correlates. Brain 129: 564-583.

53. Wagner AD, Shannon BJ, Kahn I, Buckner RL (2005) Parietal lobe contributions to episodic memory retrieval. Trends Cogn Sci 9: 445-453.

54. Fletcher PC, Frith CD, Baker SC, Shallice T, Frackowiak RS, et al. (1995) The mind's eye-precuneus activation in memory-related imagery. Neuroimage 2 195-200.

55. Honey G, Bullmore E, Sharma T (2000) Prolonged reaction time to a verbal working memory task predicts increased power of posterior parietal cortical activation. Neurolmage 12: 495-503.

56. Sommer M, Döhnel K, Sodian B, Meinhardt J, Thoermer C, et al. (2007) Neura correlates of true and false belief reasoning. Neuroimage 35: 1378-1384.

57. Kircher TT, Senior C, Phillips ML, Benson PJ, Bullmore ET, et al. (2000) Towards a functional neuroanatomy of self-processing: Effects of faces and words. Brain Res Cogn Brain Res 10: 133-144.

58. Cavanna AE, Trimble MR (2006) The precuneus: A review of its functional anatomy and behavioural correlates. Brain 129: 564-583

59. Gong G, He Y, Concha L, Lebel C, Gross DW, et al. (2009) Mapping anatomical connectivity patterns of human cerebral cortex using in vivo diffusion tensor imaging tractography. Cereb Cortex 19: 524-536.

60. Hagmann P, Cammoun L, Gigandet X, Meuli R, Honey CJ, et al. (2008) Mapping the structural core of human cerebral cortex. PLoS Biol 6: e159.

61. Margulies DS, Vincent JL, Kelly C, Lohmann G, Uddin LQ, et al. (2009) Precuneus shares intrinsic functional architecture in humans and monkeys. Proc Nat Acad Sci 106: 20069-20074.

62. DeCarli C, Massaro J, Harvey D, Hald J, Tullberg M, et al. (2005) Measures of brain morphology and infarction in the Framingham Heart Study: Establishing what is normal. Neurobiol Aging 26: 491-510.

63. Hedden T, Gabrieli JD (2004) Insights into the ageing mind: A view from cognitive neuroscience. Nat Rev Neurosci 5: 87-96.

64. Apostolova LG, Steiner CA, Akopyan GG, Dutton RA, Hayashi KM, et al. (2007) Three-dimensional gray matter atrophy mapping in mild cognitive impairment and mild Alzheimer disease. Arch Neurol 64: 1489-1495.

65. Dickerson B, Bakkour A, Salat D, Feczko E, Pacheco J, et al. (2009) The cortical signature of Alzheimer's disease: Regionally specific cortical thinning relates to symptom severity in very mild to mild $A D$ dementia and is detectable in asymptomatic amyloid-positive individuals. Cereb Cortex 19: 497-510.

66. Bakkour A, Morris JC, Dickerson BC (2009) The cortical signature of prodroma $A D$ : Regional thinning predicts mild AD dementia. Neurology 72: 1048-1055.

67. Hamalainen A, Tervo S, Grau-Olivares M, Niskanen E, Pennanen C, et al. (2007) Voxel-based morphometry to detect brain atrophy in progressive mild cognitive impairment. Neurolmage 37: 1122-1131.

68. Reiman EM, Caselli RJ, Yun LS, Chen K, Bandy D, et al. (1996) Preclinical evidence of Alzheimer's disease in persons homozygous for the epsilon 4 allele for apolipoprotein E. N Engl J Med 334: 752-758.

Citation: Chey J, Justin Kim M, Stern Y, Shin M, Byun HS, et al. (2016) Neural Substrates of Reserve Observed in a Non-Demented Aging Population. J Alzheimers Dis Parkinsonism 6: 294. doi: 10.4172/2161-0460.1000294 